\title{
Diferencias por sexo en la intimidación escolar y la resiliencia en adolescentes
}

\author{
Maribel Romero Bosa'; https://orcid.org/0000-0003-1319-7976 \\ Maria Cristina Cuevas Bohórquez'; https://orcid.org/0000-0002-3546-9605 \\ Christian Ferney Parra Olarte'; https://orcid.org/0000-0001-7485-8550 \\ Jennifer Katherine Sierra Malaver¹; https://orcid.org/0000-0002-0704-0676
}

\begin{abstract}
Resumen
El objetivo de investigación es establecer las diferencias por sexo entre la intimidación escolar y la resiliencia en adolescentes de instituciones educativas públicas de Bogotá. Se realizó un diseño de investigación no experimental transversal descriptivo-comparativo, se seleccionaron 354 participantes con edades entre 12 y 18 años. Se aplicaron la Escala de Resiliencia (Wagnild \& Young, 1993) y el Cuestionario de Intimidación Escolar Abreviado CIE - A (Moratto, Cárdenas, \& Berbesí, 2012). Los resultados muestran que existen diferencias significativas entre hombres y mujeres en la manifestación de la intimidación escolar y en las consecuencias asociadas a la salud mental; con respecto a la resiliencia, se encontraron diferencias significativas en la ecuanimidad, entendida como la habilidad de responder de manera equilibrada ante eventos estresantes. Se concluye la necesidad de un abordaje diferencial de la intimidación escolar desde la resiliencia para la creación de programas que disminuyan el fenómeno.
\end{abstract}

Palabras claves: Adolescencia; intimidación escolar; resiliência.

\section{Sex differences in adolescents in Bullying at school and resilience}

\begin{abstract}
The purpose of this research is to establish sex differences between resilience and bullying in adolescents. Transversal descriptive-comparative non experimental design was applied. 354 participants were selected with ages between 12 to 18 years old. Wagnild and Young Resilience scale (1993) and Cuestionario de Intimidación Escolar Abreviado CIE - A Abreviado (Moratto, Cárdenas, \& Berbesí, 2012), were applied. Results showed that differences studied in these groups are related to bullying expression and mental health consequences. Also Resilience showed sex differences only in the Equanimity Scale; it refers to a balanced way response to stressing events. It is necessary to design differential programs based on bullying from resilience approach to decrease the phenomenon.
\end{abstract}

Keywords: Adolescence; bullying at school; resilience.

\section{Diferenças por sexo na intimidação escolar e a resiliência em adolescentes}

\section{Resumo}

O objetivo da pesquisa é estabelecer as diferenças por sexo entre a intimidação escolar e a resiliência em adolescentes de instituições educativas públicas de Bogotá. Realizou-se um modelo de pesquisa não experimental transversal descritivo-comparativa, selecionaram-se 354 participantes com idades entre 12 e 18 anos. Aplicou-se a Escala de Resiliência (Wagnild \& Young, 1993) e o Questionário de Intimidação Escolar Abreviado CIE - A (Moratto, Cárdenas, \& Berbesí, 2012). Os resultados mostram que há diferenças significativas entre homens e mulheres na manifestação da intimidação escolar e nas consequências associadas à saúde mental; com respeito à Resiliência, encontrou-se diferenças significativas na equanimidade, entendida como a habilidade de responder de maneira equilibrada ante eventos estressantes. Conclui-seque há necessidade de uma abordagem diferencial da intimidação escolar da resiliência para a criação de programas que diminuam o fenômeno.

Palavras-chave: adolescência; intimidação; resiliência.

1 Universidad Cooperativa de Colombia - Bogotá - Colombia; maribel.romerob@campusucc.edu.co; maria.cuevasb@campusucc.edu.co; christparraolarte@gmail.com; Jennifer.sierram@campusucc.edu.co 


\section{Introducción}

La violencia en la escuela es un problema cotidiano que va en aumento. Por tanto, ha despertado el interés a nivel mundial (Bringiotti, Krynveniuk, \& Lasso, 2004). Debido a lo anterior, Elgar y cols. (2015) realizaron un estudio transeccional entre 2003 y 2011, en el cual administraron dos encuestas internacionales para medir la violencia en adolescentes; la encuesta de Salud Escolar (GSHS) y Comportamiento en salud de niños en edad escolar (HBSC) a estudiantes con edades de 11 a 16 años, en 79 países de África, Asia, Europa y América entre ellos Colombia, se encontró que el $30 \%$ de los encuestados reportaron ser víctimas de intimidación escolar y frecuentemente están involucrados en agresiones físicas $10,7 \%$ hombres y $2,7 \%$ de las mujeres. Por consiguiente, se evidencia la prevalencia del maltrato entre adolescentes. Según Garaigordobil y Oñederra (2010) se presenta en todos los países y afecta a todas las clases sociales.

Este fenómeno se presenta en diversos contextos entre ellos el acoso escolar (bullying), acoso laboral (mobbing) y acoso a través de Internet (ciberbullying), siendo expresiones de violencia interpersonal que se han convertido en un problema de salud pública (García-López, Irurtia, Caballo, \& Díaz-Castela, 2011). Esta dinámica relacional, afecta tanto a hombres como mujeres de manera diferencial. De acuerdo con lo anterior Olweous (1998); Rayzabal y Sanz (2014); Elgar y cols. (2015) plantean una tendencia en el sexo masculino a estar más expuestos a ataques físicos desde los primeros años de vida y en la educación básica secundaria en comparación con las jóvenes. Por su parte, Cerezo (2008) brinda una explicación desde una perspectiva Social y Cultural, en la cual el hombre recibe mayor aceptación de la agresividad por considerarlo un rasgo de masculinidad mientras que en la mujer se espera la conducta de sumisión.

Además, existen diferencias en la manera en que ambos sexos expresan la hostilidad; como lo plantea Cerezo (2008) los hombres emplean regularmente el ataque físico mientras que las niñas el ataque verbal y expresan la agresión de manera indirecta en conductas de aislamiento social y exclusión de su grupo de pares (Olweus, 1998). De igual manera (Reyzabal \& Sanz, 2014) afirman que las jóvenes son una baja estadística debido a que no denuncian sus experiencias de maltrato en la escuela. En contraste con los hombres quienes en mayor número se han visto involucrados en conductas de agresión dirigidas tanto a hombres como mujeres, es decir asumen roles de agresor y víctima en el acoso escolar; es de destacar que estas diferencias pueden estar determinadas por factores biológicos, sociales y medioambientales (Olweus, 1998).

Por consiguiente, la presente investigación se centró en establecer las diferencias de sexo en la Intimidación escolar y la Resiliencia, en estudiantes de octavo y noveno grado de educación secundaria. Se pretende contribuir a la comprensión del fenómeno con el fin de diseñar programas en Resiliencia para la disminución del acoso escolar, entendida la Resiliencia como una alternativa que promueve la adaptación y el afrontamiento en situaciones adversas (Wagnild \& Young, 1993) en este caso de la intimidación escolar. Las investigaciones llevadas a cabo por Olweous (1998); Reyzabal y Sanz (2014); Elgar y cols. (2015) reportan diferencias en las manifestaciones comportamentales de la intimidación escolar en cuanto a hombres y mujeres Así mismo Garaigordobil y Aliri (2013) refieren que la utilidad del análisis por sexo se valida en función de las consecuencias del acoso entre hombres y mujeres. Más aún, se quiere suplir un vacío de conocimiento con respecto a la resiliencia y sus diferencias por sexo en razón a que existe poca evidencia empírica y de esta manera aportar una aproximación conceptual al diseño de programas basados en resiliencia desde una mirada diferencial que beneficie a los actores sociales involucrados y a la sociedad en general.

\section{Diferencias de sexo en la intimidación escolar}

Es de aclarar, que en el estudio de la violencia escolar se usan diferentes términos indiferenciados como bullying, matoneo o acoso, en este estudio se precisa utilizar el concepto de intimidación escolar propuesto por (Olweus, 1997, 1998; Salmivalli, 2010) quienes plantean que es una comportamiento intencional, repetitivo en el que se expone a un estudiante que no puede defenderse a humillaciones, burlas, agresiones físicas y situaciones de exclusión, por parte de un grupo u otro compañero. En consecuencia, este tipo de interacción entre iguales puede desencadenar problemas mentales y de conducta disímiles tanto en hombres como en mujeres.

Como evidencia de lo expuesto con antelación, se realizó un estudio en Estados Unidos con el fin de conocer las características sociodemográficas y sus correlatos psiquiátricos de los participantes que fueron víctimas de intimidación escolar con respecto al sexo entre 2001 y 2002, se identificó que la prevalencia de este comportamiento de intimidación fue significativamente mayor en hombres $(8,5 \%)$ que en mujeres $(4,2 \%)$. Los análisis multivariados de regresión logística indicaron fuertes asociaciones con trastornos psiquiátricos y adictivos en ambos sexos. Además, el acoso escolar en las mujeres aumenta la probabilidad de desencadenar trastornos de control de impulsos y de tipo afectivo en comparación con los hombres (Hoertel, Le Strat, Lavaud, \&Limosin, 2012; Felipe-Castaño, León-Del Barco, \& Fajardo, 2013). I Igualmente (García-López \&cols., 2011) señalan la co-ocurrencia entre ansiedad social (Zhou, Liu, Niu, Sun, \& Fan, 2017) y depresión.

De modo similar en Granada (España) se desarrolló una investigación que tuvo como objetivo analizar y establecer los índices de Victimización, Bullying y Conducta Violenta y su posible relación con el género, residencia habitual y práctica de Actividad Física de adolescentes de 13 a 17 años, en la que se encontraron diferencias significativas con relación al sexo, los resultados contrastan con la teoría, los hombres son en mayor medida agresores y observadores, en comparación con las mujeres quienes son 
víctimas con alta frecuencia, aunque lo perciben en menor medida (Ortega Aznar, Ruz, Cortes, \& Martínez, 2015). A su vez, las mujeres que acosan a sus compañeros, tienden a usar estrategias indirectas y relacionales, a diferencia de los hombres que usan formas más directas de agresión (Navarro, Larrañaga, \& Yubero, 2011; Björkqvist, Lagerspetz, \& Kaukiainen, 1992). Por último, no se encontraron diferencias significativas en ambos sexos en cuanto a la agresión verbal (Björkqvist, Österman, \& Kaukiainen, 1992)

\section{Resiliencia}

Para empezar, la resiliencia presenta una dificultad conceptual por la falta de consenso y definiciones que no logran abarcar la totalidad del mismo (Grotberg, 1995; Munist \& cols., 1998; Becoña, 2006). En términos generales, la resiliencia se refiere a la capacidad universal que posibilita a un individuo o comunidad, expuestos a condiciones de riesgo, se sobrepongan ante situaciones adversas a través de estrategias de resolución de problemas, comunicación asertiva y el manejo adecuado de los pensamientos y sentimientos con el fin de reafirmar la capacidad de recuperación (Grotberg, 1995; Munist \& cols., 1998)

Por ende, Luthar, Cicchetti y Becker (2000); Masten y Tellegen (2012) definen resiliencia como un conjunto de procesos dinámicos que facilitan la adaptación positiva en contextos de adversidad. Por lo tanto, su naturaleza puede variar en el tiempo y con las circunstancias, una persona puede ser resiliente en un periodo de la vida y en otro no. En otras palabras, no es un estado definitivo y estable (Garrido \& De Pedro, 2005). Adicionalmente Hernández de Frutos y Del Olmo (2014) la consideran en función de la presencia de factores protectores personales, sociales, familiares e institucionales que permiten afrontar las tensiones de vida. Es de agregar, la influencia de diferencias individuales en el proceso de adaptación exitosa ante situaciones difíciles o personas poco vulnerables que se desarrollan adecuadamente a lo largo de su vida. (Garrido \& De Pedro, 2005; Rutter, 1993; Masten \&Tellegen, 2012) En suma, "La resiliencia está por construirse, para ser inventada por cada uno, en función de sí mismo y de su contexto" (Garrido \& De Pedro, 2005, p. 112)

\section{Relación entre Resiliencia e Intimidación Escolar}

En México se llevó a cabo un estudio en educación secundaria con fin de proponer la Resiliencia como estrategia terapéutica para combatir el bullying en 29 adolescentes con edades entre 15 y 17 años, de nivel socioeconómico bajo que asumen el rol de agresor. Entre los hallazgos se mejoraron las relaciones y la violencia entre pares a través de los componentes en resiliencia tales como la tolerancia, el respeto, empatía, afrontamiento y actitud positiva (Mazadiego, Vega, \& Reboredo, 2015). Así mismo, con el ánimo de reducir la prevalencia del acoso en escuelas del noreste del Reino Unido, se han adelantado intervenciones encaminadas a prevenir esta problemática y reducir sus efectos educando a las víctimas acerca de la resiliencia como estrategia para afrontar el acoso. En este estudio participaron 168 estudiantes, se encontró que los adolescentes no tienen el suficiente conocimiento de cómo superar el acoso y desarrollar la resiliencia (Garner \& Boulton, 2016)

En conclusión, el abordaje del acoso escolar debe incluir la Resiliencia, dada su efectividad en la disminución de la intimidación escolar (Mazadiego, Vega, \& Reboredo, 2015; Garner \& Boulton, 2016; Zhou \& cols., 2017). Por su parte, Garaigordobil y Aliri (2013) consideran que el análisis según el sexo es indispensable con el fin de establecer medidas educativas más adecuadas ante la creciente expresión de la agresión en niños, adolescentes y jóvenes, aunque se requiere mayor investigación al respecto.

\section{Método}

\section{Diseño y participantes}

Investigación no experimental, diseño transeccional descriptivo-comparativo (Hernández, Fernández, \& Baptista, 2014) en 354 estudiantes de octavo y noveno grado entre los 12 y 18 años; el 48, 57 \% mujeres y el 51, 43\% hombres; de estratos uno, dos y tres, en cuatro instituciones educativas públicas de las localidades de Barrios Unidos, San Cristóbal, Bosa y Puente Aranda, de la ciudad de Bogotá, se seleccionaron a través de muestreo no probabilístico de tipo intencional, donde la elección de los participantes la realiza el investigador, teniendo en cuenta los individuos más representativos según los temas de investigación o que cuenten con la experiencia apropiada en la materia (Coolican, 2005).

\section{Instrumentos}

Escala de Resiliencia para adolescentes de Wagnild y Young (1993) validada en Perú por (Castilla, Caycho, Shimabukuro, Valdivia, \& Torres-Calderón, 2014) se utilizó este instrumento debido a que no se cuentan con pruebas adaptadas y estandarizadas para el contexto colombiano. Fue diseñada para medir el grado de resiliencia individual, una puntuación mayor a 147 indican mayor capacidad de resiliencia; entre 121-146, moderada resiliencia; y valores menores a 121 , escasa resiliencia. Esta escala cuenta con25 ítems de tipo Likert con siete alternativas de respuesta entre 1 (Totalmente en desacuerdo) y 7 (Totalmente de acuerdo) y son calificados positivamente, con una puntuación máxima posible de 175 y una mínima de 25.

La medida de adecuación muestral KMO es 0.919, considerada como excelente y la prueba de Esfericidad de Barlett presenta $p<0.00$, es decir que existen intercorrelaciones lo suficientemente pequeñas entre las variables. 
El análisis de componentes principales identificó cuatro componentes con valores Eigenvalue que varían de 1.244 a 7.969. Las dimensiones del instrumento: a) La ecuanimidad; b) Perseverancia; c) Confianza y Sentirse bien solo y d) Aceptación de uno mismo:

Cuestionario Breve para Detectar intimidación escolar (Moratto, Cárdenas, \& Berbesí, 2012). Se adaptó el cuestionario de intimidación escolar CIE-A de Cuevas (2008, citado por Moratto \& cols., 2012) diseñado para el contexto colombiano. Se propone una escala abreviada de 36 ítems dirigido a estudiantes entre los 8 y los 18 años que evalúe la magnitud y características del fenómeno, debido a que relevante contar con instrumentos de corta duración y fácil comprensión logrando mayor eficiencia en su aplicación y obteniendo resultados similares a versiones extensas (Moratto \& cols., 2012); el cual mide a) la situación de victimización por intimidación (física, verbal, social y de coacción); b) la sintomatología de ansiedad, depresión, estrés postraumático y los efectos sobre autoestima, estas dos dimensiones se califican con siempre, casi siempre y nunca y, c) la intimidación por parte de quienes responden a las situaciones de intimidación donde los ítems se responden entre sí y no.

Para determinar la estructura conceptual se realizó un Análisis factorial exploratorio a través del método de componentes principales, con un $\mathrm{KMO}=0,894$ y la prueba de esfericidad de Bartlett significativa $X=5092,917, p<$ 0,000. Así mismo, los tres componentes explican el 55,8 de la varianza. Con respecto a la confiabilidad del instrumento: La victimización por intimidación, obtuvo un alfa de Cronbach de 0,87. El segundo componente, sintomatología de ansiedad, depresión, estrés postraumático y efectos sobre autoestima una consistencia interna de a 0,89. Finalmente, la intimidación por respondientes, recogió variables sobre formas de violencia por parte de las personas que realizan acciones de intimidación escolar a otros pares un alfa de Cronbach de 0,837 (Moratto \& cols., 2012)

\section{Procedimiento}

Se realizó solicitud de autorización a las instituciones educativas y trámite de consentimientos informados para los padres de los estudiantes participantes de investigación; se llevó a cabo la aplicación del instrumento Escala de Resiliencia para adolescentes de Wagnild y Young (1993) validada en el Perú por (Castilla \& cols., 2014) y Cuestionario Breve para Detectar intimidación escolar (Moratto \& cols., 2012) validado y estandarizado para Colombia, en los horarios de clase, se garantizó que fueran respondidos de manera individual y se atendieron las inquietudes con respecto al diligenciamiento de los instrumentos, se les hizo énfasis en la confidencialidad, luego se analizaron los datos a través de estadística descriptiva e inferencial con el fin de contrastar la hipótesis de investigación con respecto a las diferencias por sexo y por último, la discusión de resultados.

\section{Análisis de Resultados}

Se utilizó el software SPSS. 23, se realizaron estadísticos descriptivos e inferenciales para establecer las diferencias por sexo, se hizo la prueba de normalidad Kolmogorov-Smirnov y la prueba no paramétrica $U$ de Mann Whitney y la prueba t para muestras independientes que permiten el análisis de las diferencias entre hombres y mujeres con relación a la Intimidación Escolar y la Resiliencia.

\section{Resultados}

Respecto a los resultados de las escalas de Intimidación escolar se puede afirmar que la puntuación promedio en todas las escalas es superior en el grupo de los hombres en comparación con las mujeres.

Tabla 1. Descriptivos Cuestionario Breve para detectar la Intimidación Escolar (CIE A-ABREVIADO) (Morato, Cárdenas, y Berbesi, 2012) para hombres y mujeres.

\begin{tabular}{|c|c|c|c|c|c|}
\hline Sexo & & $\begin{array}{l}\text { Situación de victimización } \\
\text { por intimidación (Física, } \\
\text { Social y de Coacción) }\end{array}$ & $\begin{array}{l}\text { Intimidación } \\
\text { por parte de } \\
\text { respondientes }\end{array}$ & $\begin{array}{l}\text { Sintomatología de Ansiedad, } \\
\text { Depresión, EPT y efecto } \\
\text { sobre autoestima. }\end{array}$ & $\begin{array}{c}\text { Factor } \\
\text { General de } \\
\text { Intimidación } \\
\text { Escolar }\end{array}$ \\
\hline \multirow{4}{*}{ Hombre } & $M$ & 3,29 & 1,86 & 8,47 & 13,61 \\
\hline & DE & 2,735 & 2,347 & 2,805 & 4,139 \\
\hline & Mín & 0 & 0 & 0 & 1 \\
\hline & Máx & 13 & 12 & 12 & 27 \\
\hline \multirow{4}{*}{ Mujer } & $M$ & 2,52 & 0,83 & 7,37 & 10,72 \\
\hline & $\mathrm{DE}$ & 3,207 & 1,328 & 2,967 & 3,371 \\
\hline & Mín & 0 & 0 & 0 & 3 \\
\hline & Máx & 22 & 8 & 12 & 25 \\
\hline
\end{tabular}

Nota: $\mathrm{M}=$ promedio; $\mathrm{DE}=$ desviación estándar; $M$ in=rango mínimo; $M a x=$ rango máximo. 
Tabla 2. Descriptivos Escala de Resiliencia de Wagnild \& Young (1993) validada por Castilla y cols. (2014) para hombres y mujeres.

\begin{tabular}{cccccc}
\hline \multirow{3}{*}{ Sexo } & & \multicolumn{1}{c}{$\begin{array}{c}\text { Confianza y sentirse } \\
\text { bien sólo }\end{array}$} & Perseverancia & Ecuanimidad & $\begin{array}{c}\text { Aceptación de } \\
\text { uno mismo }\end{array}$ \\
\cline { 2 - 6 } Hombre & M & 44,77 & 24,42 & 27,48 & 34,23 \\
\cline { 2 - 6 } & DE & 6,545 & 3,438 & 4,243 & 2,576 \\
\cline { 2 - 6 } & Min & 21 & 12 & 15 & 23 \\
\hline \multirow{3}{*}{ Mujer } & Máx & 60 & 30 & 36 & 38 \\
\cline { 2 - 6 } & ME & 44,16 & 24,16 & 26,21 & 2,793 \\
\cline { 2 - 6 } & Mínimo & 7,340 & 4,097 & 4,844 & 23 \\
\hline
\end{tabular}

Nota: $\mathrm{M}=$ promedio; $\mathrm{DE}=$ desviación estándar; Min=rango mínimo; $M$ ax= rango máximo.

Tabla 3. Prueba No paramétrica U Mann-Whitney para Diferencias en Intimidación Escolar y Resiliencia entre hombres y mujeres

\begin{tabular}{|c|c|c|c|c|}
\hline Instrumentos & Factores & U de Mann-Whitney & Z & Sig. asintótica (bilateral) \\
\hline \multirow[t]{4}{*}{ CIE A-Abreviado } & Victimización por intimidación & 11489 & $-4,078$ & 0 \\
\hline & Intimidación por respondientes & 11226,5 & $-4,557$ & 0 \\
\hline & $\begin{array}{l}\text { Sintomatología Ansiedad, } \\
\text { Depresión, ETP y efectos en la } \\
\text { Autoestima }\end{array}$ & 11885,5 & $-3,63$ & 0 \\
\hline & Factor General de Intimidación escolar & 8494,5 & $-7,224$ & 0 \\
\hline \multirow[t]{3}{*}{ Escala de Resiliencia } & Perseverancia & 15125,5 & $-0,185$ & 0,853 \\
\hline & Ecuanimidad & 12885,5 & $-2,56$ & 0,01 \\
\hline & Aceptación de uno mismo & 13907,5 & $-1,484$ & 0,138 \\
\hline
\end{tabular}

Como se observa los hombres y las mujeres presentan puntuaciones similares en todas las escalas de Resiliencia, teniendo altas puntuaciones en Aceptación de uno mismo $(\mathrm{H}=34,23$ y $\mathrm{M}=34,56)$ y Ecuanimidad $(\mathrm{H}=27,48$ y $M=26,21)$.

Teniendo en cuenta los resultados en la validación del supuesto de normalidad, se procedió aplicar la Prueba $U$ Mann-Whitney para dos muestras independientes, se puede afirmar que existen diferencias estadísticamente significativas entre hombres y mujeres con respecto a las puntuaciones del cuestionario de Intimidación Escolar (Sig Bilateral $0.00 ; p<0,05)$.

Existen diferencias significativas entre hombres y mujeres en Resiliencia en la dimensión Ecuanimidad ( $Z=$ $-2,560 ; p=0,01<0,05)$, Debido a lo anterior se procedió a comprobar la hipótesis unilateral que los hombres son más ecuánimes que las mujeres, según los resultados se puede afirmar que la puntuación promedio de los hombres es estadísticamente mayor en comparación con las mujeres $(Z=$ $-2,560 ; p=0,005<0,05)$,

Teniendo en cuenta los resultados en la validación del supuesto de normalidad $(Z=0,200 ; p<0,05)$ y el supuesto de homogeneidad valorado a partir de la Prueba de Levene $(F=2,519 ; p<0,05)$ en la Dimensión de Confianza y sentirse bien del instrumento de Resiliencia se aplicó la Prueba $T$ para dos muestras independiente asumiendo varianzas iguales. Se puede decir que no existen diferencias significativas entre hombres y mujeres $(t=-0,819 ; p<0,05)$. 
Tabla 4. Prueba t para muestras independientes para Diferencias en Resiliencia entre hombres y mujeres.

\begin{tabular}{|c|c|c|c|c|c|c|}
\hline & & $\mathrm{T}$ & $\mathrm{Gl}$ & Sig. (bilateral) & $\begin{array}{l}\text { Diferencia de } \\
\text { medias }\end{array}$ & $\begin{array}{l}\text { Diferencia de } \\
\text { error estándar }\end{array}$ \\
\hline $\begin{array}{l}\text { Confianza y } \\
\text { sentirse bien } \\
\text { sólo }\end{array}$ & $\begin{array}{l}\text { Se asumen } \\
\text { varianzas } \\
\text { iguales }\end{array}$ & $-0,81$ & 348 & 0,414 & $-0,608$ & 0,742 \\
\hline
\end{tabular}

\section{Discusión y Conclusiones}

Esta investigación pretende comparar las diferencias de acuerdo con el sexo entre los factores de resiliencia e intimidación escolar de los estudiantes de noveno y octavo grado de instituciones educativas públicas de la ciudad de Bogotá; dentro de los hallazgos los hombres están involucrados con mayor frecuencia en contraste con las mujeres en conductas de intimidación escolar, acorde con como lo refieren autores como (Olweous, 1999; Rayzabal \& Sanz 2014; (Elgar \& cols., 2015) A su vez (Chaux, 2013) en un estudio realizado en la ciudad de Bogotá.

En lo que respecta a la resiliencia, los hombres y las mujeres presentan puntuaciones similares en todas las escalas de Resiliencia, presentando en ambos grupos altas puntuaciones en Aceptación de uno mismo $(H=34,23$ y $M=$ $34,56)$ y Ecuanimidad $(H=27,48$ y $M=26,21)$, es decir, los jóvenes muestran que creen en sí mismos, son independientes, y reconocen tanto sus debilidades como sus fortalezas y frente a la adversidad asumen una actitud de tranquilidad (Wagnild \& Young, 1993).

Con relación a las comparaciones por sexo en la intimidación escolar existen diferencias estadísticamente significativas entre hombres y mujeres en Situación de victimización, Intimidación por parte de agresores, y en el Factor General de Intimidación Escolar (Sig Bilateral 0.00; $p<0,05$ ) Con respecto a lo anterior, Navarro y cols. (2011); Björkqvist y cols. (1992); Cerezo (2008) han encontrado que las mujeres que hacen parte de comportamientos de intimidación, tienden a usar estrategias indirectas y relacionales, a diferencia de los hombres que usan formas más directas de agresión. Una de las explicaciones del fenómeno la plantea (Cerezo, 2008) quien arguye un origen social y cultural de los roles sociales asumidos por hombres y mujeres.

Por otro lado, en lo que se refiere a los síntomas relacionados con la ansiedad, depresión y manifestaciones de estrés postraumático y efectos sobre la autoestima, este resultado va en concordancia con lo que plantean (Hoertel \& cols., 2012) en lo referente a las diferencias entre hombres y mujeres en cuanto a la conducta de intimidación escolar y la prevalencia de trastornos psiquiátricos con efectos significativos de acuerdo con el sexo, estos autores consideran que las mujeres que han realizado conductas de acoso tienen una mayor probabilidad de desencadenar trastornos del control de impulsos y de tipo afectivo en contraste con los hombres. En conclusión, se deben tener en cuenta estas características diferenciales para el diseño y evaluación de programas de promoción, prevención e intervención con el propósito de disminuir las consecuencias del acoso escolar entre pares.

En consideración con la Resiliencia los resultados indican que existen diferencias estadísticamente significativas entre hombres y mujeres con respecto a las puntuaciones en la dimensión Ecuanimidad $(Z=-2,560 ; p<0,05)$, el cual se refiere al asumir la adversidad con calma y aprender de las experiencias de vida, este es un aspecto importante a tener en cuenta, ya que diferencialmente tanto hombres como mujeres cuentan con recursos o fortalezas para afrontar los eventos estresantes. Aunque se observó en los datos recolectados que los hombres tienen puntuaciones más altas que las mujeres.

De igual forma, esta dimensión es acorde con la definición de Seligman y Csikszentmihalyi (2000) de Resiliencia, quienes la asumen como la habilidad personal para dar cuenta de las adversidades que implican una actitud optimista frente a las situaciones desafiantes del contexto en el cual se desenvuelven los estudiantes, siendo un amortiguador de padecimientos tanto mentales como físicos.

Es de resaltar, que no se han llevado a cabo estudios sobre las diferencias por sexo en la resiliencia, por lo tanto, se está supliendo un vacío de conocimiento en la literatura y se sugiere mayor investigación al respecto.

\section{Referencias}

Becoña, E. (2006). Resiliencia: definición, características y utilidad del concepto. Revista de Psicopatología y Psicología Clínica, 11(3), 125-146. Recuperado: 2 ago. 2016. Disponible: http://aepcp.net/ arc/01.2006(3).Becona.pdf

Bringiotti, M. I.; Krynveniuk, M.; Lasso, S. (2004). Las múltiples violencias de la "violencia" en la escuela: desarrollo de un enfoque teórico y metodológico integrativo. Paidéia, 14(29) 313-325. https://dx.doi.org/10.1590/S0103-863X2004000300007

Björkqvist, K.; Österman, K.; Kaukiainen, A. (1992) The Development 
of Direct and Indirect Aggressive Strategies in Males and Females. In: Björkqvist, K.; Niemelä, P. (Orgs.), Of mice and women: Aspects of female aggression (pp. 51-64). San Diego, CA, US: Academic Press.

Björkqvist, K.; Lagerspetz, K.; Kaukiainen, A. (1992). Do girls manipulate and boys fight? Developmental trends in regard to direct and indirect aggression. Aggressive Behavior, 18, 117-127

Castilla, H.; Caycho, T.; Shimabukuro, M.; Valdivia, A.; TorresCalderon, M. (2014). Análisis exploratorio de la Escala de Resiliencia de Wagnild y Young en una muestra de adolescentes, jóvenes y adultos universitarios de Lima Metropolitana. Revista de Psicología de Arequipa, 4(1), 80-93

Cerezo, F. (2008). Agresores y víctimas del bullying. Desigualdades de género en la violencia entre escolares. Información Psicológica, 94, 49-59. Recuperado: 5 ago. 2016. Disponible: http://www.sc.ehu. es/ptwgalam/art_completo/2008/Informacio\%20Psicologica94.pdf

Coolican, H. (2005). Métodos de Investigación y estadística en Psicología. México Manual Moderno.

Chaux, E. (2013). Violencia escolar en Bogotá: avances y retrocesos en 5 años. Documento de Trabajo EGOB, 5. Bogotá: Escuela de Gobierno Alberto Lleras Camargo. Universidad de los Andes.

Elgar F. J.; McKinnon, B.; Walsh S. D.; Freeman J.; Donnelly P. D.; Gaspar de Matos, M.; Gariepy, G.; Aleman-Diaz, A. Y.; Pickett, W.; Molcho, M.; Currie, C. (2015). Structural determinants of youth bullying and fighting in 79 countries. Journal of Adolescent Health, 57(6), 643-650. Recuperado: 14 abr. 2017. Disponible: http:// dx.doi.org/10.1016/j.jadohealth.2015.08.007.

Felipe-Castaño, E.; León-Del-Barco, B.; Fajardo, F. (2013). Perfiles psicopatológicos delos participantes en situaciones deacosoescolar en educación secundaria. Psicología Conductual, 21(3),475-490. Recuperado: 14 abr. 2017. Disponible: http://Search.Proquest. Com/Docview/1476261054?Accountid=44394

Garaigordobil, M.; Oñederra, J. A. (2010). La violencia entre iguales. Madrid: Pirámide.

Garaigordobil, M.; Aliri, J. (2013). Ciberacoso ("Cyberbullying”) en el País Vasco: diferencias de sexo en víctimas, agresores y observadores. Psicología Conductual, 21(3), 461-474. Recuperado: 5 nov. 2016. Disponible: http://Search.Proquest.Com/ Docview/1476261032?Accountid $=44394$

García-López, L. J.; Irurtia, M. J.; Caballo, V. E.; Díaz-Castela, M. M. (2011). Ansiedad social y abuso psicológico. Behavioral Psychology/Psicología Conductual, 19, 223-236. Recuperado: 20 sep. 2016. Disponible: http://search.proquest.com/docview/92774 3563 ? accountid $=44394$

Garnerl W.; Boulton, M., J. (2016). Adolescent's unambiguous knowledge of overcoming bullying and developing resilience.
Journal of Occupational Therapy, Schools \& Early Intervention,9(2) 199-207. Recuperado: 14 abr. 2017. Disponible: http://dx.doi.org/1 $0.1080 / 19411243.2016 .116276$

Garrido, V.; De Pedro, F. (2005). Educar para la resiliencia. Un cambio de mirada en la prevención de situaciones de riesgo social.Revista Complutense de Educación, 16(1) 107-124. Recuperado: 18 sep. 2016. Disponible: https://revistas.ucm.es/index.php/RCED/article/ view/RCED0505120107A

Grotberg, E. (1995). A guide to promoting resilience in children: streng the ning the human spirit. Bernard Van Leer Foundation. Disponible: http://www.bibalex.org/search4dev/files/283337/115519.pdf

Hernández, R.; Fernández, C.; Baptista, P. (2014) Metodología de la investigación (6a. ed.). México: McGraw-Hill Education.

Hernández de Frutos, T.; Del Olmo, N. (2014). Factores de riesgo y protección/resiliencia en el acoso escolar adolescente análisis longitudinal. Revista Internacional de Sociología,72(3) 583-608. Recuperado: 20 mai. 2016. Disponible: http://revintsociologia. revistas.es/index.php/revintsociologia/article/view/597/620 doi:10.3989/ris.2013.05.07

Hoertel, N.; Le Strat Y.; Lavaud, P.; Limosin, F. (2012). Gender effects in bullying: results from a national simple. Psychiatry Research, 200, 921-927. Recuperado: 10 abr. 2017. Disponible: http://dx.doi. org/10.1016/j.psychres.2012.03.036.

Luthar, S. S.; Cicchetti, D.; Becker, B. (2000). The construct of resilience: a critical evaluation and guidelines for future work. Child Development, 71(3), 543-562.

Masten., A. S.; Tellegen (2012).Resilience in developmental psychopathology: contributions of the Project competence longitudinal study. Development and Psychopathology, 24, 345361. doi: 10.1017/S095457941200003X

Mazadiego, M. T.; Vega, O.J.; Reboredo, J. F. (2015). Resiliencia como estratégia terapêutica antibullying. Revista Electrónica de Psicología de Iztacala, 18(3), 1241-1260. Disponible: http://www. revistas.unam.mx/index.php/repi/article/view/52694

Moratto, N.; Cárdenas, N.; Berbesí, D. (2012). Validación de un cuestionario breve para detectar intimidación escolar. Revista CES Psicología, 5(2), 70-78. Recuperado: 25 mai. 2016. Disponible: http://www.scielo.org.co/pdf/cesp/v5n2/v5n2a06.pdf

Munist, M.; Santos, H.; Kotliarenco, M.; Suárez Ojeda, E.; Infante, F.; Grotberg, E. (1998). Manual de identificación y promoción de la resiliencia en niños y adolescentes. Organización Mundial de Salud. Disponible: http://www.1.paho.org/hq/dmdocuments/2009/ Resilman.pdf

Navarro, R.; Larrañaga, E.; Yubero, S. (2011). Bullying-victimization problems and aggressive tendencies in spanish secondary schools students: the role of gender stereotypical traits. Social Psychology 
of Education: an International Journal, 14(4), 457-473. doi:http:// dx.doi.org/10.1007/s11218-011-9163-1

Ortega, F.; Aznar, J. M. V.; Ruz, R. P.; Cortes, A. J. P.; Martínez, A. M. (2015). Conductas agresivas y de bullying desde la perspectiva de actividad física, lugar de residencia y género en adolescentes de Granada/Aggressive and bullying from the perspective of physical activity, place of residence and sex behavior among adolescents in Granada. Revista Complutense De Educación, 26(3), 527-542. Recuperado: 6 abr. 2017. Disponible: http://search.proquest.com/ docview/1733145162? accountid=44394.

Olweus, D. (1997). Bully/victimproblems in school: Facts and intervention. European Journal of Psychology of Education, 12(4), 497-510. Disponible: http://www.episcenter.psu.edu/sites/default/ files/news/Olweus\%20(1997)\%20Bully-victim\%20problems\%20 in\%20school.pdf

Olweus, D. (1998). Conductas de acoso y amenaza entre escolares. España: Ediciones Morata.

Reyzabal, M. V.; Sanz, A. I. (2014). Resiliencia y acoso escolar La fuerza de la educación. Madrid: La Muralla.
Rutter (1993). Resilience: some conceptual considerations. Journal of Adolescent Health, 14(8) 626-631.

Salmivalli, C. (2010). Bullying and the peer group: A review. Aggression and Violent Behavior, 15, 112-120. Recuperado: 7 nov. 2016. Disponible: http://njbullying.org/documents/bullyingandpeergrroup. pdf

Seligman, M. E. P., Csikszentmihalyi, M. (2000). Positive psychology: $\mathrm{Na}$ introduction. American Psychologist, 55(1), 5-14. Recuperado: 25 sep. 2016. Disponible: http://dx.doi.org/10.1037/0003066X.55.1.5

Wagnild, G. M.; Young, H. M. (1993). Development and psychometric evaluation of the Resilience Scale. Journal of Nursing Measurement, 1, 165-178.

Zhou, K., Liu, Q., Niu, G., Sun, X., \& Fan, C. (2017) Bullying victimization and depression in Chinese children: A moderated mediation model of resilience and mindfulness, Personality and Individual Differences, 104, 137-142, http://dx.doi.org/10.1016/j. paid.2016.07.040. 